\title{
Systemic risk contributions ${ }^{1}$
}

\author{
Xin Huang, Hao Zhou and Haibin Zhu
}

\section{Introduction}

The recent global financial crisis has led to a re-examination and reform of the international regulatory framework, and most remarkably to the introduction of macroprudential regulation. Against this background, this paper proposes a consistent framework for measuring systemic risk and decomposing it into contributions from individual banks. Our systemic risk measure can be interpreted economically as an insurance premium that covers distressed losses in a banking system, which is the concept of a risk-neutral market price, assuming that such an insurance market exists and functions properly. Within the same framework, the systemic importance of each bank (or a group of banks) can be properly defined as its marginal contribution to the hypothetical distress insurance premium (DIP) of the whole banking system. This approach allows us to study the time variation and cross section of the systemic risk contributions of large and complex US financial institutions (LCFIs). Our metric can be applied using only publicly available information for large banking organisations.

We applied this approach to the 19 bank holding companies (BHCs) covered by the US Supervisory Capital Assessment Program (SCAP) - commonly known as the macro stress test - during the period from January 2004 to December 2009. However, unlike the SCAP, our analysis did not rely on any confidential, supervisory, or proprietary information or data. Our findings suggest that the systemic risk indicator stood at its peak of around $\$ 1.1$ trillion in March 2009 and has since fallen to about $\$ 300$ billion - the level reached in January 2008. A bank's contribution to the systemic risk indicator appears to be linearly related to its default probability but highly nonlinear with respect to institution size and its asset correlation with the rest of the banking system.

More importantly, we can rank the systemic importance of LCFIs in the US banking sector. Based on our measure since the summer of 2007, Citigroup has remained the largest contributor to systemic risk, the contributions of Bank of America and Wells Fargo have risen, and that of JPMorgan Chase has declined. The relative contributions to systemic risk from both consumer banks and regional banks seem to have increased somewhat since 2009, possibly because of the deteriorating situation in the commercial real estate and consumer credit sectors, which typically lag the business cycle. Overall, our analysis suggests that size is the dominant factor in determining banks' systemic risk contributions, but size does not change significantly over time, at least within a reporting quarter. The obvious time variations in the marginal contributions are driven mostly by the risk-neutral default probability and equity return correlations. In essence, the systemic importance of each institution is jointly determined by the size, default probability and asset correlation of all institutions in the portfolio.

Finally, our measure of the systemic importance of financial institutions noticeably resembles the SCAP result. Based on the data through 31 December 2008, the 19 banks' contributions to the systemic risk indicator are mostly in line with the SCAP estimate of losses under an

1 The note is an abridged version of "Systemic risk contributions", Finance and Economics Discussions Series, Federal Reserve Board, 2011-08, 2011.

2 Xin Huang is with the University of Oklahoma, Hao Zhou is with the Federal Reserve Board, and Haibin Zhu was with the BIS when the paper was presented at the conference. He is now at JPMorgan. 
adverse economic scenario as released on 9 May 2009, with an R-square of 0.62 . Not surprisingly, the two results are not identical, because the SCAP estimate is based on statistical expected losses but our systemic risk measure also contains a risk premium component. In particular, Goldman Sachs, Citigroup and JPMorgan Chase would be viewed as contributing more to systemic risk by our method (from a risk premium perspective) than by the SCAP results, while Bank of America and Wells Fargo would be viewed as more risky by the SCAP results (from an expected loss perspective) than by our method.

\section{Methodology}

\subsection{Constructing the systemic risk indicator}

The systemic risk indicator, a hypothetical insurance premium against catastrophic losses in a banking system, was constructed from real-time publicly available financial market data. The two key default risk factors, the probability of default (PD) of individual banks and the asset return correlations among banks, were estimated from CDS spreads and equity price co-movements, respectively.

The PD measure used in this approach was derived from single-name CDS spreads. A CDS contract offers protection against default losses of an underlying entity; in return, the protection buyer agrees to make constant periodic premium payments. The CDS market has grown rapidly in recent years, and the CDS spread is considered superior to the bond spread or the loan spread as a measure of credit risk. Under the simplifying assumptions of a flat term structure of the risk-free rate and a flat default intensity term structure, the one-year risk-neutral PDs of individual banks can be derived from CDS spreads in a straightforward manner (Huang, Zhou and Zhu (2009)).

Systemic risk in a financial sector is in essence a joint default event of multiple large institutions, which is captured by the correlations of observable equity returns. At a more fundamental level, such a correlation structure is driven by the common movements in underlying firms' asset values. The common movements can be due to the business cycle, interbank business linkages or shift in market sentiment that affects the valuation of bank assets simultaneously. We measured the asset return correlation by the equity return correlation, as the equity market is the most liquid financial market and can incorporate new information on an institution's default risk in a timely way. The standard approach is to use the so-called historical correlation, which is based on the past year of daily return data.

Based on the inputs of the key credit risk parameters - PDs, LGDs, correlations and liability weights - the systemic risk indicator can be calculated by simulation (Huang, Zhou and Zhu (2009)). In short, to compute the indicator, we first construct a hypothetical debt portfolio that consists of the total liabilities (deposits, debts, and others) of all banks. The indicator of systemic risk, effectively weighted by the liability size of each bank, is defined as the insurance premium that protects against the distressed losses of this portfolio. Technically, it is calculated as the risk-neutral expectation of portfolio credit losses that equal or exceed a minimum share of the sector's total liabilities (10\% in our case).

\subsection{Identifying systemically important banks}

For the purpose of macroprudential regulation, it is important not only to monitor the level of systemic risk for the banking sector but also to understand the sources of risk in the financial system, ie to measure the marginal contributions of each institution. This information is especially useful considering the reform effort of the financial regulations across the globe, with the main objective of charging additional capital for systemically important banks and supporting a resolution regime for these banks. 
Following the standard measures of risk, including VaR, ES and the DIP used in this study, the total risk can be usefully decomposed into a sum of marginal risk contributions (Huang, Zhou and Zhu (2010)). Each marginal risk contribution is the expected loss from that subportfolio, conditional on a large loss for the full portfolio. The additivity property of the decomposition results - ie the fact that the systemic risk of a portfolio equals the marginal contribution from each subportfolio - is extremely important from an operational perspective. Whereas the macroprudential approach focuses on the risk of the financial system as a whole, in the end regulatory and policy measures are introduced at the level of individual banks. Our approach, therefore, allows a systemic risk regulator to easily link the regulatory capital assessment with risk contributions from each institution.

The most closely related approach is the CoVaR method proposed by Adrian and Brunnermeier (2009). CoVaR looks at the VaR of the whole portfolio conditional on the VaR of an individual institution. In other words, the focus of CoVaR is to examine the spillover or correlation effect from one bank's failure to the whole system, but CoVaR underplays the importance of institutional size by design. By comparison, our definition of DIP is along the same line, but DIP focuses on the loss of a particular bank (or bank group) conditional on the system being in distress. Nevertheless, a major disadvantage of CoVaR is that it can be used only to identify systemically important institutions but cannot appropriately aggregate the systemic risk contributions of individual institutions.

Another alternative is the MES proposed by Acharya, Pedersen, Philippon and Richardson (2010). MES looks at the expected loss of each bank conditional on the whole portfolio of banks performing poorly. Again, in comparison, MES is similar to our DIP measure in that both focus on each bank's potential loss conditional on the system being in distress exceeding a threshold level, and both are coherent risk measures. They differ slightly in the sense that the extreme condition is defined by the percentile distribution in the MES setting but by a given threshold loss of the underlying portfolio in the case of DIP. Also, the probabilities in the tail event underpinning MES are normalised to sum to 1; these probabilities are not normalised for DIP. The more important difference is that MES is calculated based on equity return data, while our DIP measure is based mainly on the CDS data. Compared with equity return data, CDS data are better and purer sources of default risk information.

\section{Empirical application}

We applied the methodology described in Section 2 and examined the systemic risk in the US banking system consisting of the 19 banks covered by the SCAP, commonly known as the macro stress test. Using these banks as an example, we first reported the systemic risk indicator for these institutions as a group and then analysed the systemic importance of individual banks.

Figure 1 reports the time variation of the DIP. The systemic risk indicator for the US banking system was very low at the beginning of the financial and credit crises. For a long period before the collapse of two Bear Stearns hedge funds in early August 2007, the DIP for the list of 19 SCAP banks was merely one-half of 1 percentage point (or less than $\$ 5$ billion). The indicator then moved up significantly, reaching the first peak when US bank regulators arranged for Bear Stearns to be acquired by JPMorgan Chase on 16 March 2008. The situation then improved significantly in April and May 2008 owing to strong intervention by major central banks. Things changed dramatically in September 2008 with the failure of Lehman Brothers. Market panic and increasing risk aversion pushed up the price of insurance against distress in the banking sector. The DIP shot up and hovered in the range of $\$ 500$ billion to $\$ 900$ billion. One week before the stock market reached the bottom, the systemic risk indicator peaked at around \$1.1 trillion. Since the release of the SCAP result in 
early May 2009, the DIP has come down quickly and has returned to the pre-Lehman level of \$300-400 billion.

Figure 1

Systemic risk indicator of the 19 US SCAP banks
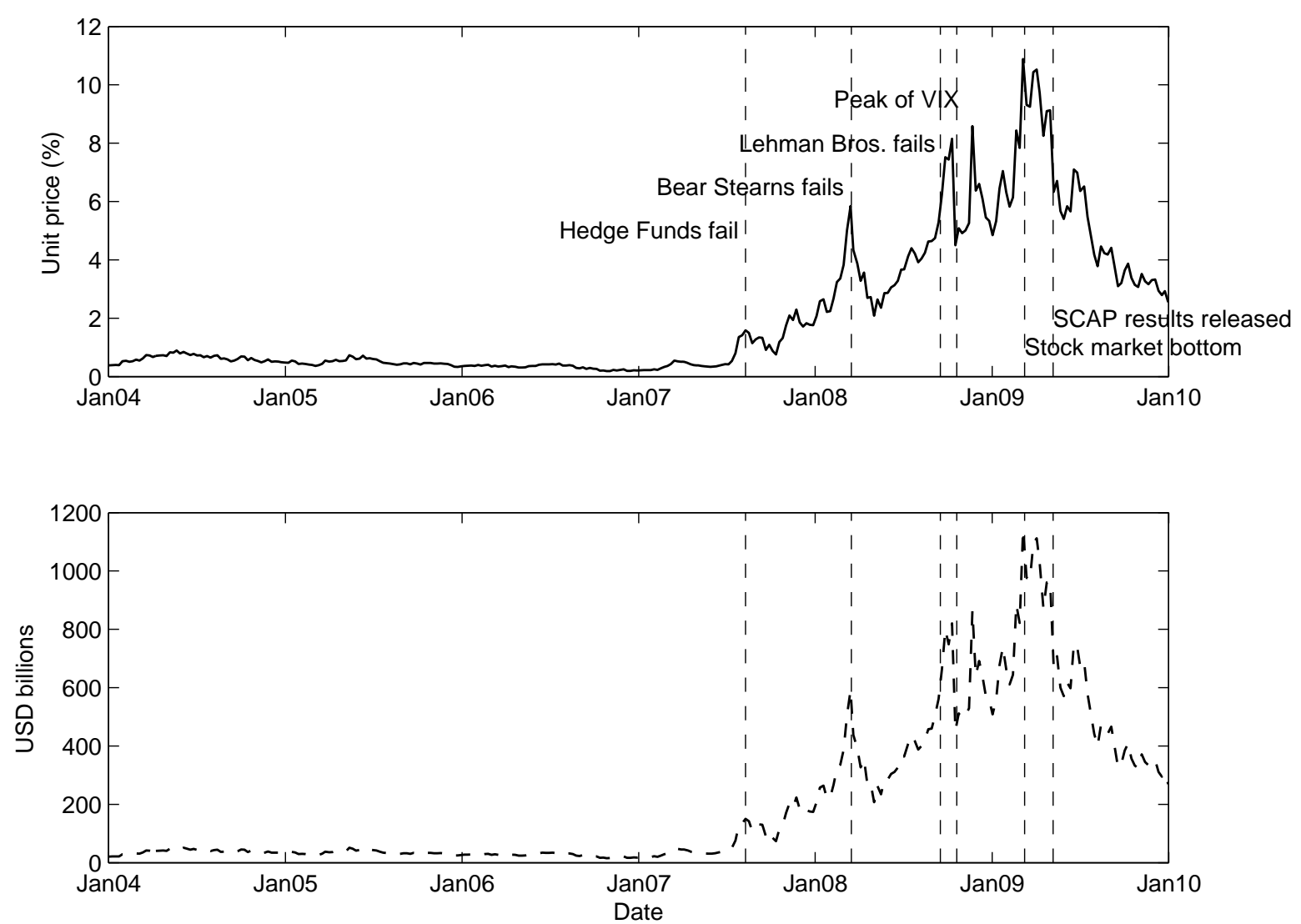

Notes: The figure plots the systemic risk indicator for the SCAP banks, defined as the price for insuring against financial distresses (at least 10\% of total liabilities in the banking system are in default). The price is shown as the cost per unit of exposure to these liabilities in the upper panel and is shown in dollar terms in the lower panel.

Using the methodology described in Section 2, we calculated the marginal contributions of each group of banks to the systemic risk indicator, both in level terms and in percentage terms. Figure 2 shows that, based on our measure after the summer of 2007, Bank of America and Wells Fargo increased their systemic risk contributions, Citigroup remained the largest contributor, and JPMorgan Chase reduced its marginal contribution. Recall that Wells Fargo acquired Wachovia, and Bank of America acquired Merrill Lynch, during the height of the financial crisis. Figure 2 also reports the systemic risk contributions of other banks, which are grouped into four categories. ${ }^{3}$ The relative contributions to the systemic risk indicator from both consumer banks and regional banks seem to have increased somewhat since

3 The four categories are as follows: (1) investment banks (Goldman Sachs and Morgan Stanley); (2) consumer banks (GMAC and American Express); (3) regional banks (US Bancorp, Capital One, PNC Financial, SunTrust, BB\&T, Regions Financial, Fifth Third and KeyCorp); and (4) processing banks (Bank of New York Mellon, State Street and Northern Trust). Bank of America, Citigroup, JPMorgan Chase and Wells Fargo are listed as individual large complex financial institutions. 
2009, possibly because of the worsening situations in the commercial real estate and consumer credit sectors, which typically lag the business cycle.

Figure 2

\section{Marginal contributions to systemic risk by each group of banks}
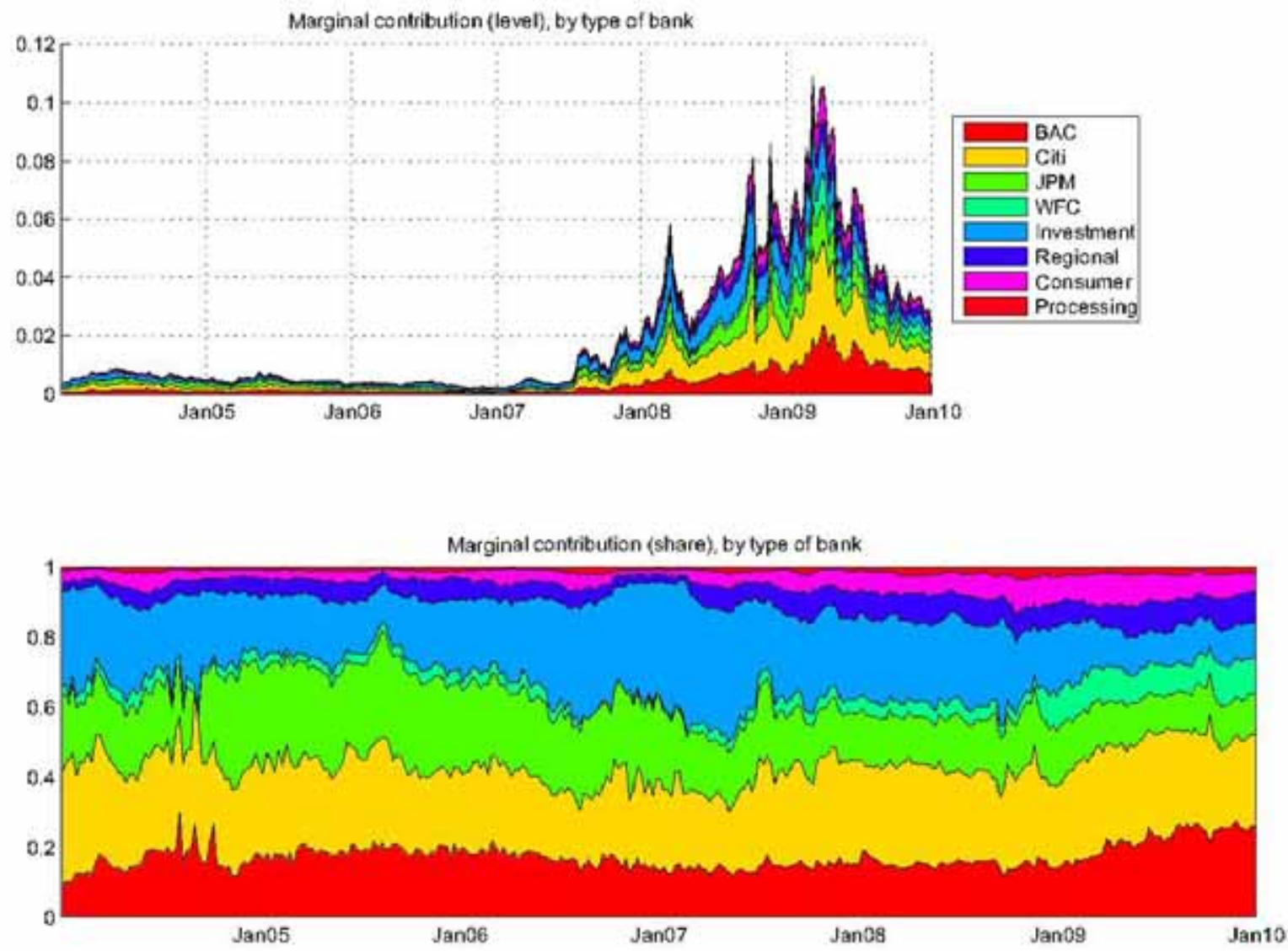

Notes: The figure shows the marginal contribution of each bank or banking group to the systemic risk indicator, the distress insurance premium in unit cost term. The contribution is shown in level terms in the upper panel and as a percentage of the total risk in the lower panel.

The above findings support the case for distinguishing between microprudential and macroprudential perspectives of banking regulation: the failure of individual banks does not necessarily contribute to the increase in systemic risk. Size, correlation and the interactions between the determinants play important roles. The nonlinear effect is more visible in a hypothetical calibration exercise examining the relationship between our measure of systemic risk contributions and an institution's size (total liability), (risk-neutral) default probability, and (average) historical correlation (Figure 3). The relationship looks roughly linear for default probability but highly nonlinear with respect to size and, to a lesser degree, correlation. In fact, when the bank size is below $10 \%$ of the total portfolio, the slope of the systemic importance with respect to size is very flat; but when the size is beyond $10 \%$, the contribution to systemic risk shoots up almost vertically. An intuitive reason is that, when a bank is too big, its failure is considered a systemic failure by definition. This consideration may indicate a desirable maximum size of the large complex financial institutions, which, by limiting the systemic risk, could provide a societal benefit. The relationship between systemic importance and correlation shows a similar nonlinear pattern but is less dramatic. In other words, systemic importance is a joint effect of an institution's size, leverage, and concentration and is highly nonlinear. 
Figure 3

\section{Relationship between systemic risk contribution and bank size, PD and correlation}
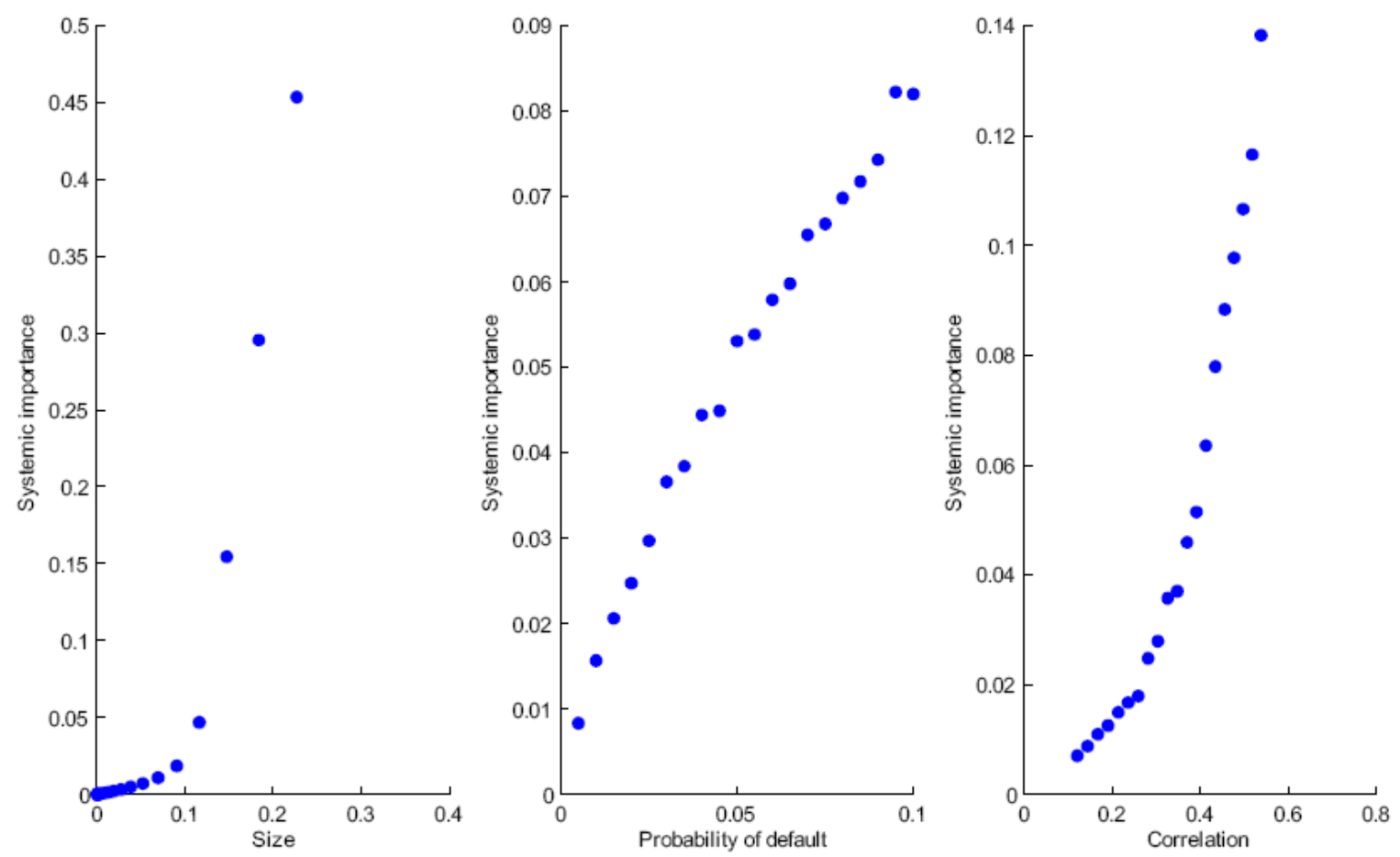

Notes: This figure plots a hypothetical calibration exercise based on a group of 20 banks, with average LGD of 0.55 and distress threshold 10\%. For the impact of size (left panel), PD is 0.02 and correlation is $20 \%$; for the impact of PD (middle panel), PD changes from 0.005 to 0.1 ; for the impact of correlation (right panel), the loading coefficient in a one-factor model ranges between 0.2 and 0.96 .

We can further compare different measures of systemic importance with the SCAP estimate of losses under an adverse economic scenario as released in May 2009 by the Federal Reserve (2009). The left-hand panel of Figure 4 suggests that, based on the data through 31 December 2008, the 19 banks' contributions to our DIP systemic risk indicator are largely in line with the SCAP estimate of losses, with an R-square of 0.62. Note that the SCAP estimate is based on statistical expected losses but our systemic risk measure also contains a risk premium component. As a result, the exact ranking of systemic importance can be different. For instance, Goldman Sachs, Citigroup and JPMorgan Chase would be viewed as contributing much more to systemic risk by our method from a market risk premium perspective than by the SCAP results, while Bank of America and Wells Fargo would be viewed as more risky by the SCAP from an expected default loss perspective than by our method.

The middle panel shows that MES weighted by Tier 1 capital has a higher correlation with SCAP expected losses, with an R-square of 0.71. Relative to SCAP, MES considers Bank of America, JPMorgan Chase and Goldman Sachs more risky and Wells Fargo and Citigroup less risky. The right panel shows that CoVaR in dollar terms has a similar correlation with the SCAP results, with an R-square of 0.63. Compared with SCAP, CoVaR ranks JPMorgan Chase, MetLife, and Goldman Sachs as more risky but Citigroup and Bank of America as less risky. 
Figure 4

\section{Comparing systemic risk measures: DIP, MES and CoVaR versus SCAP results}
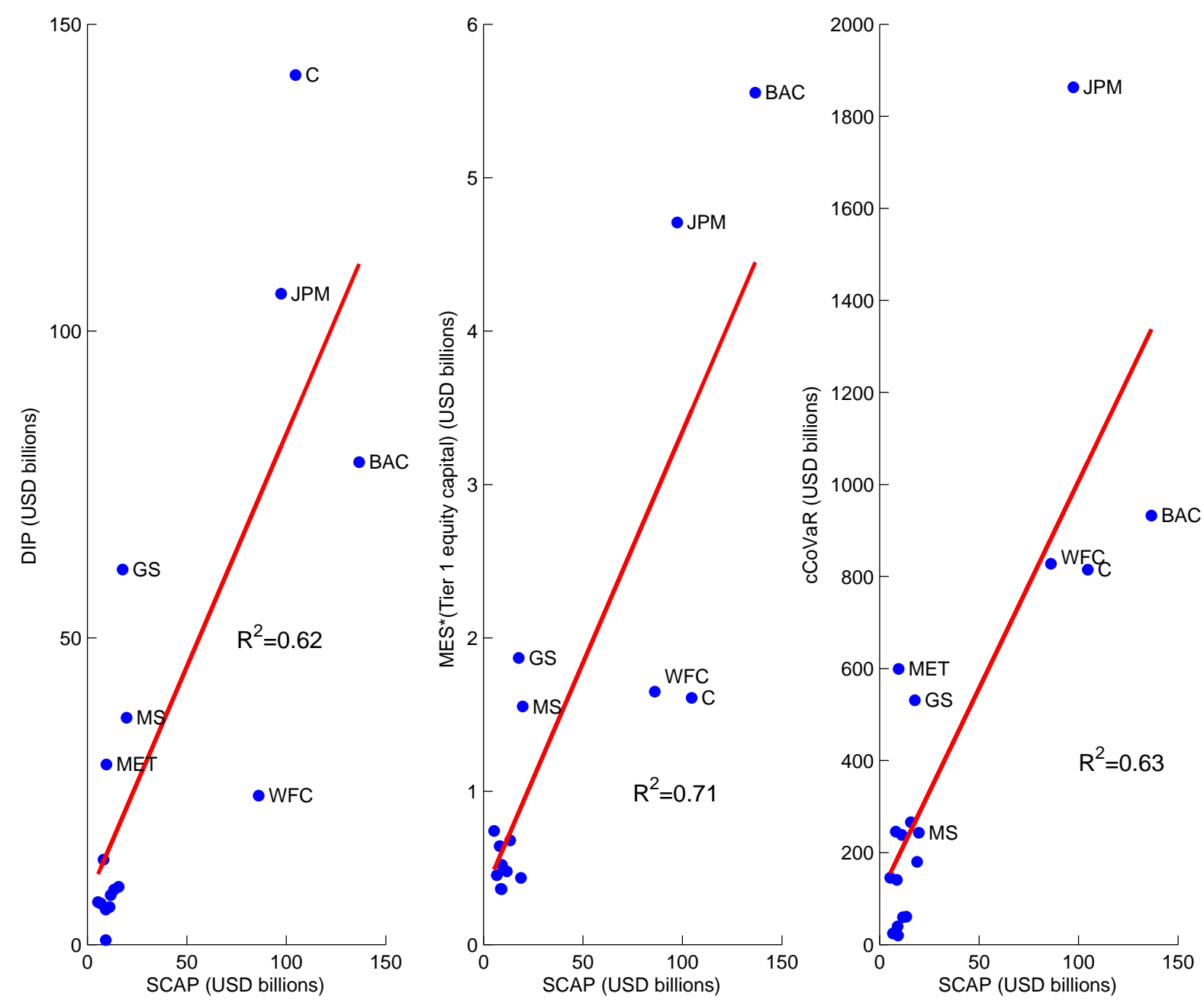

Notes: This figure compares three systemic risk measures: distressed insurance premium (DIP), marginal expected shortfall (MES) weighted by bank's Tier-1 capital, and conditional value-at-risk (CoVaR) in dollar terms. These measures are compared to the SCAP stress test result on the 19 largest US BHCs for the fourth quarter of 2008.

\section{Policy implications}

In this paper, we advocate a methodology to measure the systemic importance of individual banks and their marginal contributions to a distressed insurance premium that relies only on publicly available information. We applied this methodology to the 19 banks covered by the SCAP, or stress test programme. Our results have several important policy implications.

First, our analysis provides useful inputs for the ongoing discussion of the imposition of capital surcharges on systemically important financial institutions (SIFIs). The 2007-09 global financial crisis has led the international community of supervisors and regulators to reform the regulatory framework to ensure that a crisis on this scale never again happens. As an important part of the global initiatives, there is a general consensus that SIFIs need to set aside an additional capital buffer. In practice, the Swiss regulator has announced a plan to impose total capital requirements of as high as $19 \%$ on the two largest Swiss banks, of which systemic surcharges account for 6 percentage points. Similarly, the Chinese regulator has 
imposed a minimum capital adequacy ratio of $11.5 \%$ for large banks, in contrast to one of $10 \%$ for small and medium-sized banks.

However, much remains to be decided regarding the definition of SIFIs and the calculation of capital surcharge for SIFIs. In this paper, we show that the systemic importance of financial institutions depends on their size, correlation and PD, which is highly consistent with views widely shared among regulators and supervisors. More importantly, the additivity property of our systemic risk contributions, as discussed in Section 2.2, makes it feasible to directly map our measures onto capital surcharges. Preliminary analysis shows a high correlation between our systemic importance measures and the US government's capital infusion into the banking system in 2008-09. Further analysis is necessary to make the mapping of our systemic risk contributions onto capital surcharges more rigorous.

Second, although the proposed DIP measure is risk-neutral, the framework can be easily extended by replacing key inputs with the regulator's confidential information or other input variables for the purpose of policy analysis. For instance, one can replace the risk-neutral PDs in our framework with objective measures of PDs and calculate the DIP on an incurredcost basis. This objective measure, by filtering out the risk premium components, can provide useful complementary information for supervisors.

Third, our systemic risk indicator is designed as a real-time signal of the systemic risk in a banking system, and cannot be interpreted directly as an early warning indicator. Indeed, the DIP measure was low in 2007 and went up rapidly as the crisis deepened. Thus users should be careful in interpreting the results. However, there are ways to incorporate our measure into an early warning system, for example, in the stress-testing exercise as illustrated in Huang, Zhou and Zhu (2009).

\section{References}

Acharya, V, L Pedersen, T Philippon and M Richardson (2010): "Measuring systemic risk", Working Paper, NYU Stern School of Business.

Adrian, T and M Brunnermeier (2009): "CoVaR", Federal Reserve Bank of New York Staff Reports, no 348, September.

Federal Reserve Board (2009): "The Supervisory Capital Assessment Program: Overview of results", May.

Huang, X, H Zhou and H Zhu (2009): "A framework for assessing the systemic risk of major financial institutions", Journal of Banking and Finance, vol 33, pp 2036-49.

(2010): "Assessing the systemic risk of a heterogeneous portfolio of banks during the recent financial crisis", Working Paper, Federal Reserve Board.

US Congress (2010): "Dodd-Frank Wall Street Reform and Consumer Protection Act", Public Document, HR 4173. 